\title{
Molecular characterization of Haemophilus parasuis ferric hydroxamate uptake $(f h u)$ genes and constitutive expression of the FhuA receptor
}

\author{
Maria Luisa DEL RíO ${ }^{a}$, Jesús NAVAS ${ }^{b}$, Ana Judith MARTíN ${ }^{\mathrm{a}}$, \\ César B. GutiérReZ ${ }^{\mathrm{a}}$, José-Ignacio RodRÍGUEZ-BARBOSA ${ }^{\mathrm{c}}$, \\ Elías F. RODRÍGUEZ FERRI ${ }^{\mathrm{a} *}$ \\ a Microbiology and Immunology Section, Department of Animal Health, \\ Faculty of Veterinary Medicine, Leon, Spain \\ ${ }^{b}$ Department of Molecular Biology, School of Medicine, Santander, Spain \\ ${ }^{c}$ Research Unit, Arrixaca University Hospital, Murcia, Spain
}

(Received 16 December 2004; accepted 20 June 2005)

\begin{abstract}
Bacteria have evolved a set of highly specialized proteins to capture iron in irondepleted environments. The acquisition and uptake of iron present in the extracellular milieu of eukaryotic organisms is indispensable for the growth and survival of microbial pathogens in the course of infection. Haemophilus parasuis is the causative agent of Glässer disease, which is responsible for considerable financial losses in pig-rearing worldwide. To gain insight into the mechanisms involved in siderophore-mediated iron uptake in $H$. parasuis, genes in the $H$. parasuis ferric hydroxamate uptake (Fhu) region were amplified in the work being reported here. As has been described in A. pleuropneumoniae, an Fhu genomic region was also present in $H$. parasuis, being composed of four potential consecutive open reading frames (ORF) designated as fhuC, fhuD, fhuB, and fhuA, respectively. By immunoblotting, using a cross-reactive polyclonal antibody raised against Actinobacillus pleuropneumoniae FhuA protein, it was demonstrated that this protein was constitutively expressed in $H$. parasuis and its level of expression was not modified under conditions of restricted iron availability. This is the first report describing the presence of the fhu genes in $H$. parasuis. Our results indicate that FhuA protein expression is not affected under ironrestricted conditions, however, it is one of the targets of the humoral immune response.
\end{abstract}

Haemophilus parasuis / ferric hydroxamate uptake / iron

\section{INTRODUCTION}

Haemophilus parasuis (H. parasuis) is a non-motile, nicotinamide adenine dinucleotide (NAD)-dependent, small pleomorphic Gram-negative rod of the family Pasteurellaceae (belonging to the $\gamma$ subclass of the class Proteobacteria). This species is the causative agent of Glässer disease, a stressassociated disease of young pigs involving fibrinous polyserositis, polyarthritis and meningitis. This infectious disease is emerging as a serious problem in the pig-rearing industry worldwide, particularly since specific-pathogen free herds have been established. It is responsible for considerable

\footnotetext{
* Corresponding author: dsaerf@unileon.es
} 
financial losses and often associated with high morbidity and mortality rates [28].

The pathogenicity of Glässer disease is largely unknown and only a few virulence factors have been described so far. As has been described in other Gram-negative microorganisms, lipopolysaccharide is responsible for endotoxic shock and its side effects [1]. Several studies have also reported that the virulence is associated with the presence of a capsule [14], certain outer membrane profiles [24, 25], fimbriae [22] or neuraminidase (sialidase) $[15,16]$.

Iron is an essential element required for bacterial growth and multiplication. In mammalian tissues, most of the iron is bound to serum proteins such as transferrin, hemoglobin and lactoferrin [17]. To gain access to these sources of iron, pathogenic bacteria use high-affinity outer membrane proteins (OMP) capable of binding serum proteins transporting iron. These OMP are therefore involved in iron uptake and in the majority of the microorganisms, they upregulate their expression under conditions of limited iron availability. Moreover, the majority of the microorganisms is also capable of producing or using high-affinity iron-binding compounds, called siderophores, which can chelate the ferric iron and thus can incorporate it through specialized OMP receptors. These receptors have been associated with the virulence of many pathogenic bacteria [10].

Morton and Williams proposed the first model of iron acquisition in $H$. parasuis [21], and showed that this bacterium could bind porcine transferrin. Later on, Charland et al. assigned this function to two potential porcine transferrin binding polypeptides, which could specifically bind iron from porcine transferrin, but not from porcine lactoferrin [7].

Four consecutive genes named fhuA, $f h u C$, fhuD, and $f h u B$ compose the E. coli Fhu region [5]. The Fhu proteins cooperate to capture siderophores, such as ferric hydroxamate, from the extracellular milieu and transport them to the cytoplasm. Ferri- chrome-loaded siderophores are first captured by the outer membrane receptor (FhuA). Then, FhuA transfers the siderophore to FhuD (periplasmic protein), which in turn translocates the siderophore ferric hydroxamate from the outer to the inner membrane. Finally, FhuB and FhuC (components of the $\mathrm{ABC}$ transporter) located in the cytoplasmic membrane, cooperate to internalize the siderophores to the cytoplasm.

The purpose of this work was to detect and characterize the $H$. parasuis fhu genes and to answer the question of whether FhuA siderophore receptor expression was regulated under iron-restricted conditions.

\section{MATERIALS AND METHODS}

\subsection{Bacterial strains and growth conditions}

The reference strains of $H$. parasuis and field isolates of the most frequent serotypes of $H$. parasuis isolated in Spain that were used in this study are listed in Table I. $H$. parasuis strains were cultured onto chocolate agar plates (BioMérieux, Barcelona, Spain) and incubated for $24 \mathrm{~h}$ at $37^{\circ} \mathrm{C}$. Iron-restricted conditions were achieved by addition of $100 \mu \mathrm{M} 2.2$ dipyridyl (Sigma, Madrid, Spain) to PPLO broth (Difco, Barcelona, Spain) supplemented with $0.025 \%$ NAD (Sigma). Actinobacillus pleuropneumoniae (A. pleuropneumoniae) serotype 7 was grown under the same culture conditions as described above and was used as a positive control in polymerase chain reaction (PCR) and immunoblotting experiments.

\subsection{Recombinant DNA techniques}

PCR amplification of the $H$. parasuis Fhu region was carried out with the Expand Long Template PCR System (Roche Diagnostics, Madrid, Spain) from genomic DNA of $H$. parasuis with different forward and reverse pairs of primer combinations that were designed on the basis of A. pleuropneumoniae homologous gene sequences 
Table I. Strains used in this study.

\begin{tabular}{|c|c|c|}
\hline Strain & Description & Source \\
\hline H. parasuis & \multicolumn{2}{|l|}{ Reference serotype } \\
\hline No.4 & Serotype 1 & Kielstein P. \\
\hline SW140 & Serotype 2 & Kielstein P. \\
\hline SW114 & Serotype 3 & Kielstein P. \\
\hline SW124 & Serotype 4 & Kielstein P. \\
\hline Nagasaki & Serotype 5 & Kielstein P. \\
\hline 131 & Serotype 6 & Kielstein P. \\
\hline 174 & Serotype 7 & Kielstein P. \\
\hline C5 & Serotype 8 & Kielstein P. \\
\hline D74 & Serotype 9 & Kielstein P. \\
\hline Н 555 & Serotype 10 & Kielstein P. \\
\hline Н 465 & Serotype 11 & Kielstein P. \\
\hline Н 425 & Serotype 12 & Kielstein P. \\
\hline 84-17975 & Serotype 13 & Kielstein P. \\
\hline $84-22113$ & Serotype 14 & Kielstein P. \\
\hline 84-15995 & Serotype 15 & Kielstein P. \\
\hline H. parasuis & Field isolates & \\
\hline Нp9121 & Serotype 1 & Spain \\
\hline Hp1521 & Serotype 3 & Spain \\
\hline Hp622 & Serotype 4 & Spain \\
\hline Hp66 & Serotype 4 & Spain \\
\hline Нp3021 & Serotype 5 & Spain \\
\hline Hр871 & Serotype 9 & Spain \\
\hline Hр39521 & Serotype 10 & Spain \\
\hline Hp624 & Serotype 12 & Spain \\
\hline Нp547 & Serotype 14 & Spain \\
\hline Нp2521 & Serotype 15 & Spain \\
\hline \multicolumn{3}{|c|}{ A. pleuropneumoniae Reference serotype } \\
\hline WF83 & Serotype 7 & Nielsen R. \\
\hline
\end{tabular}

(GenBank accession number AF351135). The isolation of DNA, PCR, recovery of DNA fragments from agarose gels, and agarose gel electrophoresis were performed using standard techniques as outlined by Sambrook et al. [26] or as recommended by the commercial suppliers (QIAGEN, Barcelona, Spain). The primers for PCR amplification and sequencing of the $H$. parasuis Fhu region are listed in Table II.

\subsection{DNA sequencing}

DNA sequencing was performed at "Sistemas Genómicos" DNA Sequencing Core Facility in Valencia (Spain) using a capillary Beckman CEQ 2000 XL sequencer in accordance with the manufacturer's instructions. Both strands of each gene included in the Fhu region were sequenced. The sequences obtained were analyzed using the BLAST computer program at the National Center for Biotechnology Information (www.ncbi.nlm.nih.gov/BLAST). The computer software package DNAMAN version 5.2 (Lynnon Biosoft), was also used for designing the primers and alignment of the sequences. To calculate the GC content, the Oligonucleotide Properties Calculator (http://www.basic.nwu.edu/biotools/oligocalc.html) was used.

\subsection{Growth conditions and preparation of protein extracts from $H$. parasuis and $A$. pleuropneumoniae from whole-cell lysates}

Whole-cell lysates from $H$. parasuis and A. pleuropneumoniae were prepared from bacterial culture grown under iron-rich and iron-poor conditions. A single colony of $H$. parasuis or A. pleuropneumoniae was inoculated into $2.5 \mathrm{~mL}$ PPLO medium and incubated overnight at $37^{\circ} \mathrm{C}$. Then, $0.5 \mathrm{~mL}$ of the overnight culture were inoculated into $4.5 \mathrm{~mL}$ PPLO medium and incubated under agitation until the cell density reached $0.3-0.4$ at $\mathrm{OD}_{660}$. To create ironrestricted conditions, $100 \mu \mathrm{M} 2.2$ dipyridyl was added to the culture medium and allowed to grow while it was shaken for $2 \mathrm{~h}$. Finally, $1.5 \mathrm{~mL}$ of bacterial cell culture were harvested by centrifugation at $11000 \times g$ for $5 \mathrm{~min}$, and the supernatant was removed. The protein concentration was estimated by the micro-BCA protein assay with a bovine serum albumin standard (Pierce, Bonn, Germany). Five microlitres of the cell suspension were mixed with $5 \mu \mathrm{L}$ of $2 \times \mathrm{SDS}$ sample buffer, boiled at $100{ }^{\circ} \mathrm{C}$ for $5 \mathrm{~min}$ and centrifuged. Samples were separated on 
Table II. Primers used in this study.

\begin{tabular}{lcc}
\hline Primers & Sequence & Source or reference \\
\hline FhuC ini & 5' AAT AGC TTC CTT TTA TTT ATT C 3' & This study \\
C For & 5' GCA ATT CGA GCA GGG TAA G 3' & {$[19]$} \\
C Rev & 5' CGG GTC GTT TGG TTT CAG G 3' & {$[19]$} \\
FhuCD F & 5' TGA AAC CAA ACG ACC GGT GAG 3' & This study \\
D For & 5' GTG AGC TTC CGT ATT TCT 3' & {$[19]$} \\
D Rev & 5' CGC CTC CGT GCA ATA ATC 3' & {$[19]$} \\
B For & 5' CTT ATT CAG CGG ATT AGC C 3' & {$[19]$} \\
FhuB 2 & 5' CGT TTT CGC CAT TAA TGA 3' & This study \\
FhuB 3 & 5' GCT TAG GTG TGC CGG TAG CAA AAT 3' & This study \\
FhuBA F & 5' AAG TTT TAT CGG TTT ACT TGT A 3' & This study \\
B Rev & 5' CAA CTA ATG TGG CGA CTA AG 3' & {$[19]$} \\
FhuB 2R & 5' TGT TAA GAA TTT TTT AAA TTG T 3' & This study \\
FhuA 2F & 5' ATT CGA CAG TCG CTT CGG CA 3' & This study \\
A For & 5' GAT GAG GTG TCG GTG GTT 3' & {$[19]$} \\
Fhu BA R & 5' GTC GGT AAG CCG TCC ACG GC 3' & This study \\
FhuA R & 5' ATC AGA GGT ATA TTC GTT TT 3' & This study \\
FhuA 3R & 5' TCT TTT AAT AGG TTT TGC AA 3' & This study \\
\hline
\end{tabular}

$12 \%$ SDS-PAGE gel at $100 \mathrm{~V}$ during $1.5 \mathrm{~h}$. A pre-stained molecular weight standard (Precision Plus Protein Dual Color Standards, BioRad, Madrid, Spain) was run in parallel on the same gel to verify the molecular weight of the proteins present in the sample.

\subsection{Immunoblotting}

SDS-polyacrilamide gel was transferred to a nitrocellulose membrane (Biorad) and immunoblotting was performed following standard procedures [26]. Briefly, non-specific binding sites of the nitrocellulose membrane were blocked by incubating the membranes for $1 \mathrm{~h}$ at room temperature in Tris saline buffer (TBS) (10 mM Tris, $150 \mathrm{mM}$ $\mathrm{NaCl}[\mathrm{pH} 7.1])$ containing $5 \%(\mathrm{w} / \mathrm{v})$ skim milk. Then, the nitrocellulose membrane was incubated overnight at $4{ }^{\circ} \mathrm{C}$ with an optimal dilution (1:1000) of a cross-reactive rabbit polyclonal antibody against $A$. pleuropneumoniae FhuA protein. This polyclonal antiserum was kindly provided by Dr GeraldF. Gerlach (Institute for Microbiology, Hannover Veterinary School, Germany) [3].
A porcine convalescent serum from $H$. parasuis serovar 5-infected pig and the same serum collected prior to infection were used in immunoblotting to demonstrate that antiFhuA antibodies were generated during the course of infection. All the polyclonal sera were incubated as primary antibody at optimal dilution, then, the membranes were washed three times with TBS-Tween 20 $0.05 \%(\mathrm{v} / \mathrm{v})$. To make sure that the addition of 2.2 dipyridyl was chelating the free iron available in the culture medium, the Transferein Binding Protein $\mathrm{B}(\mathrm{TbpB})$ protein was chosen as a marker due to the fact that its expression is up-regulated under iron restricted conditions [8]. The level of expression of the $\mathrm{TbpB}$ protein was monitored with an anti-TbpB polyclonal antibody raised against a recombinant $\mathrm{TbpB}$ fusion protein of $H$. parasuis [8]. Horseradish peroxidase (HRPO)-conjugated goat anti-rabbit IgG (whole molecule) (1:2000, Sigma) or (HRPO)-conjugated goat anti-pig IgG (whole molecule) (1:5000) was added to the membrane and incubated for $1 \mathrm{~h}$ at room temperature. Finally, the reaction was developed by 
the addition of Enhanced Chemiluminiscent substrate (Amersham Biosciences, Barcelona, Spain) to the membranes for $1 \mathrm{~min}$ [6].

\subsection{Nucleotide sequence accession number}

The nucleotide sequence of the H. parasuis serotype 5 Fhu region was deposited in the GenBank database with the accession number AY676933.

\section{RESULTS}

\section{1. fhu genes are present in $H$. parasuis}

Long template PCR amplification was carried out with primers flanking the Fhu region (FhuC ini and FhuA 3R, Tab. II), using the following PCR protocol $\left(94^{\circ} \mathrm{C} 2\right.$ ', $\left[94^{\circ} \mathrm{C} 30^{\prime},, 55^{\circ} \mathrm{C} 1\right.$ ', $\left.68^{\circ} \mathrm{C} 4^{\prime}\right] \times 30,68^{\circ} \mathrm{C}$ $8^{\prime}$ '). A 5800 base pair (bp) PCR product was obtained, containing the complete Fhu region in the $H$. parasuis serotype 5 reference strain (Fig. 1). Similar results were obtained with the remaining $H$. parasuis reference strains (data not shown). fhu genes were also detected in several serotypes of $H$. parasuis field isolates (data not shown). For sequencing the $H$. parasuis serotype 5 Fhu region, the primers listed in Table II were used.

\subsection{Characterization of the $\boldsymbol{H}$. parasuis Fhu region}

Sequence analysis of the $f h u$ PCR product revealed the presence of four potential

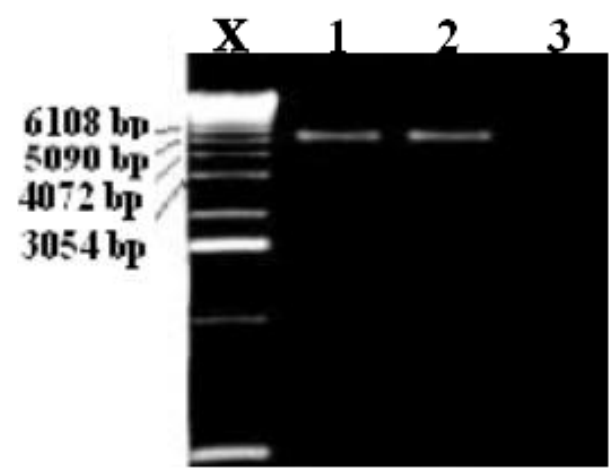

Figure 1. Presence of the $f h u C D B A$ region (5 $800 \mathrm{bp}$ ) in H. parasuis serotype 5 . The primers used were FhuC ini FhuA $3 \mathrm{R}$. Lane X, Molecular weight marker No. X, ranging from 70 to 12200 bp (Roche Diagnostics). Lane 1, $H$. parasuis; lane 2 , A. pleuropneumoniae serotype 7 (positive control); lane 3, distilled water (negative control).

open reading frames, encoding 255,318 , 651 and 696 amino acids (AA), respectively. These ORF showed almost $100 \%$ identity with the A. pleuropneumoniae fhu genes. The deduced molecular mass, gene length, and number of AA are shown in Table III. Fenograms showing the genetic distances relative to homologous proteins of other bacteria (A. pleuropneumoniae [GenBank accession number AF351135], S. Typhimurium [GenBank accession number NC_003197], and E. coli [GenBank accession number NC_000913]) are shown in Figures $2 \mathrm{a}$ and $2 \mathrm{~d}$.

Table III. Characterization of $H$. parasuis serotype 5 fhu genes and percentage of AA sequence identity of $H$. parasuis Fhu proteins with homologous proteins from other Gram-negative bacteria.

\begin{tabular}{lcccccc}
\hline Gene & Base pairs & $\begin{array}{c}\text { Amino } \\
\text { acids }\end{array}$ & $\begin{array}{c}\text { Molecular } \\
\text { mass (kDa) }\end{array}$ & \multicolumn{3}{c}{ Identity (\%) of H. parasuis with } \\
\hline fhuC & 765 & 255 & 28.5 & 100 & 51 & 53 \\
fhuD & 954 & 318 & 35.6 & 100 & 23 & 27 \\
fhuB & 1953 & 651 & 69.4 & 99 & 40 & 38 \\
fhuA & 2088 & 696 & 77.1 & 100 & 26 & 27 \\
\hline
\end{tabular}




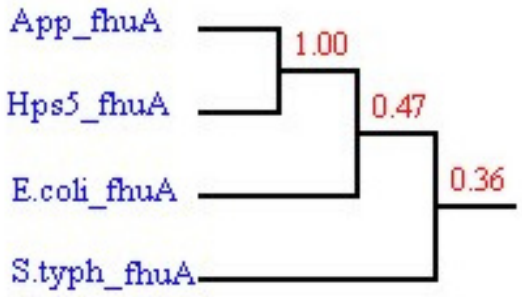

$\mathbf{a}$

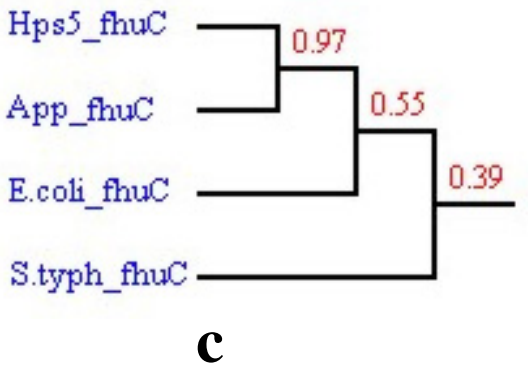

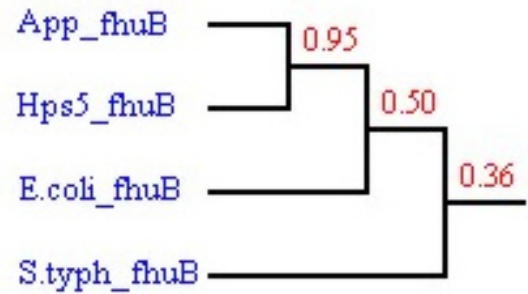

b

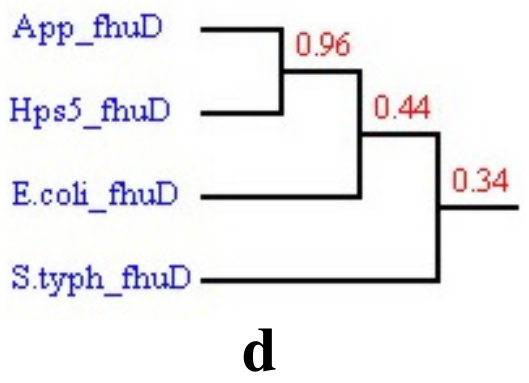

Figure 2. Fenograms showing the genetic distances between $H$. parasuis fhu genes and A. pleuropneumoniae, S. Typhimurium, and E. coli. (a) fhuA; (b) fhuB; (c) fhuC, and (d) fhuD.



Figure 3. Organization of the $H$. parasuis Fhu region showing the gene length, the primers used for amplification and the location of the Shine-Dalgarno (SD) sequences.

The four genes encoding FhuC, FhuD, FhuB and FhuA proteins are located consecutively on the chromosome, in the following order: fhuC, fhuD, fhuB and fhuA (Fig. 3), similar to what has been reported for A. pleuropneumoniae. Fur boxes were not identified along $H$. parasuis Fhu region. The nucleotide sequence of $f h u C$ includes nucleotides 1 to 765 . The start codon of $f h u D$ is ATG, located at position 765 , 

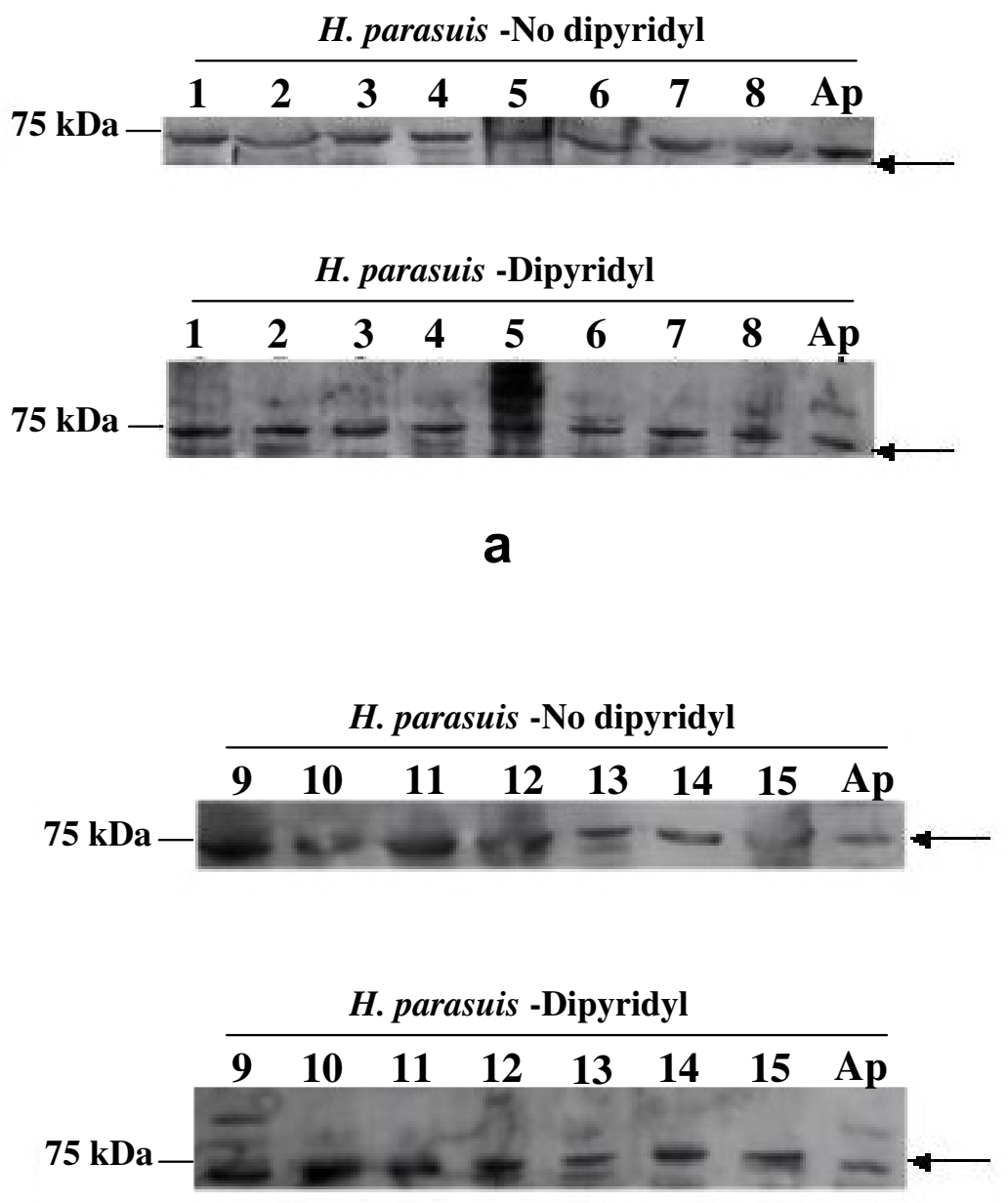

b

Figure 4. Immunoblotting using a cross-reactive polyclonal antibody against A. pleuropneumoniae FhuA protein, showing the presence of the FhuA protein in the fifteen reference strains of $H$. parasuis tested, under iron-replete and iron-restricted conditions using the iron-chelating compound 2.2 dipyridyl. (a) $H$. parasuis serotypes 1 to 8 (lanes 1-8), and A. pleuropneumoniae serotype 7 (Ap) with and without 2.2 dipyridyl. (b) H. parasuis serotypes 9 to 15 (lanes 9-15), and A. pleuropneumoniae serotype 7 (Ap) with and without 2.2 dipyridyl. The protein concentration was estimated by the micro-BCA protein assay with a bovine serum albumin standard and identical concentration of the protein was loaded in each lane.

overlapping with the stop codon of $f h u C$. A Shine-Dalgarno (SD) sequence $6 \mathrm{bp}$ was found upstream of the $f h u B$ start codon (ATG). The $f h u B$ start codon is located $8 \mathrm{bp}$ upstream of the $f h u D$ stop codon (TAA). There are $47 \mathrm{bp}$ between the $f h u B$ stop codon and the fhuA start codon, where a second SD sequence 8 bp upstream of the 


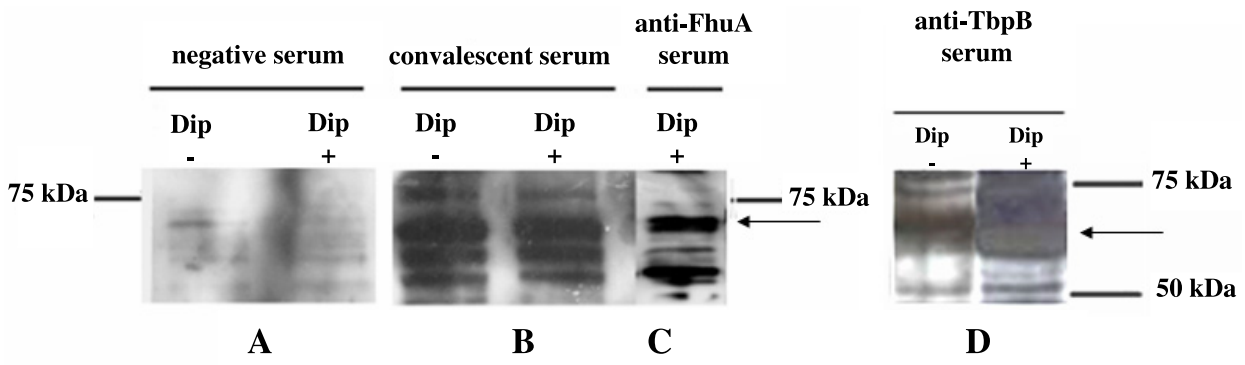

Figure 5. Whole-cell lysates of $H$. parasuis serovar 5 grown under iron-restricted or iron-repleted conditions were tested by immunoblotting with the following polyclonal anti-sera: (A) negative serum; (B) convalescent serum; (C) anti-FhuA serum, and (D) anti-TbpB serum. The protein concentration was estimated by the micro-BCA protein assay with a bovine serum albumin standard and identical concentration of the protein was loaded in each lane.

fhuA start codon was found. The GC content of the $H$. parasuis Fhu region was $44 \mathrm{~mol} \%$.

Overall, these data confirm the presence of the Fhu region involved in siderophoremediated uptake of iron in $H$. parasuis.

\subsection{H. parasuis FhuA protein is not upregulated under iron-restricted conditions}

To determine whether $H$. parasuis FhuA protein was expressed under conditions of restricted iron availability, $H$. parasuis strains were grown in an iron-replete medium and in an iron-restricted medium by the addition of 2.2 dipyridyl (final concentration $100 \mu \mathrm{M}$ ). $H$. parasuis protein extracts were run in SDS-PAGE gels and transferred onto nitrocellulose membranes. Thereafter, cross-reactive anti-FhuA polyclonal antibody against A. pleuropneumoniae FhuA protein was added and a band of an approximate molecular weight of $75 \mathrm{kDa}$ was detected in all $H$. parasuis serotypes (Figs. $4 \mathrm{a}$ and $4 \mathrm{~b}$ ). $H$. parasuis FhuA protein was expressed constitutively in all the reference strains of $H$. parasuis, and its expression was not therefore affected under iron-restricted conditions. Under these iron-restricted conditions $H$. parasuis strains (serotypes 1, 2, 5, and 7) were however capable to grow in the presence of ferrichrome as a sole source of iron (data not shown).

\subsection{FhuA induces a productive humoral immune response during the course of $H$. parasuis infection}

To assess whether FhuA was one of the targets of the humoral immune response during the course of $H$. parasuis infection, pigs were infected with $H$. parasuis and the humoral immune response was monitored by immunoblotting. Thus, sera collected from pigs prior to infection with $H$. parasuis (Fig. 5a) and convalescent sera collected from $H$. parasuis-infected pigs (Fig. 5b) were incubated with whole-cell lysates of $H$. parasuis grown under iron-restricted and iron-repleted conditions. We observed that $H$. parasuis infection stimulated a productive humoral immune response against FhuA protein (Fig. 5c). To ensure that ironrestricted conditions were achieved in our experimental setting, $H$. parasuis TbpB protein was used as control as the expression of this protein is upregulated under ironrestricted conditions. Our data indicate that FhuA protein was immunogenic and capable of stimulating anti-FhuA antibodies during the course of natural infection. 


\section{DISCUSSION}

The ability to compete for essential nutrients such as iron, which are in limited supply in the extracellular milieu of eukaryotic organisms, is a well-recognized requirement of virulent bacteria capable of inducing disease in susceptible mammalian hosts. Bacteria have evolved high affinity iron uptake systems to face environments surrounded by limited amounts of iron. Owing to this low iron availability in the host, the ability of the microorganisms to sequester iron is widely recognized as a virulence factor $[4,10]$. Mechanisms for iron acquisition involve two general categories: synthesis of siderophores and/or receptor-mediated acquisition of iron bound to host proteins [23]. Until recently, it was an open question whether $H$. parasuis could acquire iron through transferrin binding proteins or siderophores. Although, it seems clear that $H$. parasuis is capable of using porcine transferrin [7] as a source of iron for growth, scarce information has so far been available about siderophore-mediated mechanisms of iron uptake in $H$. parasuis. This work has demonstrated the presence of the Fhu region and the expression of the FhuA receptor in $H$. parasuis. These data support the hypothesis that $H$. parasuis may use ferrichrome as a source of iron. Our observations are in agreement with the data reported by other authors who claim that ferrichrome is capable to promote the growth and survival of $H$. parasuis serotype 5 [9] and A. pleuropneumoniae [20] under iron-limiting conditions.

The ferrichrome uptake system comprises four genes disposed in tandem and encoding four proteins that cooperate in the steps of uptake, translocation and transport of the siderophore to the cytoplasm of the bacteria [4]. The work reported here involved successful cloning and sequencing of the $H$. parasuis Fhu region. The presence of fhuCDBA genes clearly supports the concept that $H$. parasuis strains may count for all the necessary Fhu proteins for capturing iron from siderophores. However, these results disagree with others reported recently in the literature [20], in which it is claimed that the fhuA gene and the corresponding protein encoded were not detected in $H$. parasuis when primers, probes and polyclonal serum generated against $A$. pleuropneumoniae FhuA protein were used. The authors in question argue that the negative results obtained in their experiments might be due to the lack of similarity between the fhuCDBA sequences of $H$. parasuis and of A. pleuropneumoniae. In contrast to their results, it proved possible to demonstrate that $H$. parasuis fhu genes showed a high degree of homology (99-100\%), in comparison with A. pleuropneumoniae fhu genes (Tab. III). The GC content of the H. parasuis Fhu region is $44 \mathrm{~mol} \%$ a value that correlated well with the estimated $43 \pm$ $2 \mathrm{~mol} \%$ reported for the genome of A. pleuropneumoniae [19]. Moreover as predicted, a polyclonal anti-serum raised against A. pleuropneumoniae recombinant fusion FhuA protein cross-reacted with the H. parasuis FhuA protein. Despite the high degree of homology found between $H$. parasuis and A. pleuropneumoniae, the comparison of $H$. parasuis fhu sequences with the homologous $f h u$ sequences of members of the family Enterobacteriaceae, such as $S$. Typhimurium or $E$. coli showed only $26 \%$ homology.

The relative position of the four genes in the $H$. parasuis Fhu region was identical to that of A. pleuropneumoniae [19], in which the $f h u C$ gene is followed by the $f h u D, f h u B$ and $f h u A$ genes, whereas in $E$. coli or $S$. Typhimurium the $f h u A$ is followed by fhuC, fhuD and fhuB $[11,18]$. The different relative position of the four genes in $H$. parasuis and A. pleuropneumoniae, as compared to $E$. coli and $S$. Typhimurium $f h u$ genes, might account for the differences in the regulation of the FhuA protein expression observed in the former species. Although none of the consensus promoters were found, more work is still required to gain insight into the upstream region of the $H$. parasuis fhu $\mathrm{C}$ gene, where putative regions with promoter activity may be located. 
The presence of the FhuA receptor in all the serotypes of $H$. parasuis was also demonstrated by immunoblotting. A $75 \mathrm{kDa}$ protein was detected, with a molecular weight corresponding to the size of the FhuA protein. Its expression was constitutive and therefore independent of the iron concentration available in the culture medium. These results were in agreement with those reported for A. pleuropneumoniae FhuA protein [20]. Moreover, $H$. parasuis-infected pigs developed a humoral immune response against $H$. parasuis FhuA protein as we demonstrated by immunoblotting, indicating that the extracellular loops of this protein are exposed to the immune system during the course of infection.

However, unlike $H$. parasuis and A. pleuropneumoniae, the expression of the FhuA protein in E. coli [13] and C. jejuni [12] is upregulated under iron-restricted conditions. This positive regulation is mediated by the Fur protein $[12,27]$ but so far a Fur homologue has not been described in $H$. parasuis yet. Interestingly, and in contrast with other iron uptake mechanisms, such as transferrin binding proteins [2, 29], FhuA expression in $H$. parasuis was constitutive. As far as the authors are aware, this is the first report to show the presence of $f h u$ genes and the expression of the FhuA protein in $H$. parasuis. Further iron-binding studies and mutagenesis experiments are required with the aim of proving the relevance of these genes in the pathogenicity of H. parasuis.

\section{ACKNOWLEDGEMENTS}

We would like to express our gratitude to Dr P. Kielstein for providing us with reference strains of $H$. parasuis. This work was supported by grants AGF 99-0196 and AGL 2002-04585C02-01 GAN-ACU from the Spanish Ministry of Science and Technology.

\section{REFERENCES}

[1] Amano H., Shibata M., Takahashi K., Sasaki Y., Effects on endotoxin pathogenicity in pigs with acute septicemia of Haemophilus parasuis infection, J. Vet. Med. Sci. 59 (1997) 451-455

[2] Baltes N., Hennig-Pauka I., Gerlach G.F., Both transferrin binding proteins are virulence factors in Actinobacillus pleuropneumoniae serotype 7 infection, FEMS Microbiol. Lett. 209 (2002) 283-287.

[3] Baltes N., Tonpitak W., Hennig-Pauka I., Gerlach G.F., Actinobacillus pleuropneumoniae serotype 7 siderophore receptor FhuA is not required for virulence, FEMS Microbiol. Lett. 220 (2003) 41-48.

[4] Braun V., Iron uptake by Escherichia coli, Front. Biosci. 8 (2003) 1409-1421.

[5] Braun V., Braun M., Iron transport and signaling in Escherichia coli, FEBS Lett. 529 (2002) 78-85.

[6] Bronstein I., Voyta J.C., Murphy O.J., Bresnick L., Kricka L.J., Improved chemiluminescent western blotting procedure, Biotechniques 12 (1992) 748-753.

[7] Charland N., D'Silva C.G., Dumont R.A., Niven D.F., Contact-dependent acquisition of transferrin-bound iron by two strains of Haemophilus parasuis, Can. J. Microbiol. 41 (1995) 70-74.

[8] Del Río M.L., Gutiérrez-Martín C.B., RodríguezBarbosa J.I., Navas J., Rodríguez-Ferri E.F., Identification and characterization of the TonB region and its role in transferrin-mediated iron acquisition in Haemophilus parasuis, FEMS Immunol. Med. Microbiol. 45 (2005) 75-86.

[9] Diarra M.S, Dolence J.A., Dolence E.K., Darwish I., Miller M.J., Malouin F., Jacques M., Growth of Actinobacillus pleuropneumoniae is promoted by exogenous hydroxamate and catechol siderophores, Appl. Environ. Microbiol. 62 (1996) 853-859.

[10] Faraldo-Gomez J.D., Sansom M.S., Acquisition of siderophores in gram-negative bacteria, Nat. Rev. Mol. Cell. Biol. 4 (2003) 105116.

[11] Fecker L., Braun V., Cloning and expression of the fhu genes involved in iron (III)-hydroxamate uptake by Escherichia coli, J. Bacteriol. 156 (1983) 1301-1314.

[12] Galindo M.A., Day W.A., Raphael B.H., Joens L.A., Cloning and characterization of a Campylobacter jejuni iron-uptake operon, Curr. Microbiol. 42 (2001) 139-143.

[13] Hantke K., Braun V., Membrane receptor dependent iron transport in Escherichia coli, FEBS Lett. 49 (1975) 301-305. 
[14] Kielstein P., Rosner H., Muller W., Typing of heat-stable soluble Haemophilus parasuis antigen by means of agargel precipitation and the dot-blot procedure, Zentralbl. Veterinarmed. B 38 (1991) 315-320.

[15] Lichtensteiger C.A., Vimr E.R., Neuraminidase (sialidase) activity of Haemophilus parasuis, FEMS Microbiol. Lett. 152 (1997) 269274.

[16] Lichtensteiger C.A., Vimr E.R., Purification and renaturation of membrane neuraminidase from Haemophilus parasuis, Vet. Microbiol. 93 (2003) 79-87.

[17] Litwin C.M., Calderwood S.B., Role of iron in regulation of virulence genes, Clin. Microbiol. Rev. 6 (1993) 137-149.

[18] McClelland M., Sanderson K.E., Spieth J., Clifton S.W., Latreille P., Courtney L., Porwollik S., Ali J., Dante M., Du F., Hou S., Layman D., Leonard S., Nguyen C., Scott K., Homes A., Grewal N., Mulvaney E., Ryan E., Sun H., Florea L., Miller W., Stoneking T., Nhan M., Waterston R., Wilson R.K., Complete genome sequence of Salmonella enterica serovar Typhimurium LT2, Nature 413 (2001) 852-856.

[19] Mikael L.G., Pawelek P.D., Labrie J., Sirois M., Coulton J.W., Jacques M., Molecular cloning and characterization of the ferric hydroxamate uptake (fhu) operon in Actinobacillus pleuropneumoniae, Microbiology 148 (2002) 2869-2882.

[20] Mikael L.G., Srikumar R., Coulton J.W., Jacques M., fhuA of Actinobacillus pleuropneumoniae encodes a ferrichrome receptor but is not regulated by iron, Infect. Immun. 71 (2003) 2911-2915.

[21] Morton D.J., Williams P., Utilization of transferrin-bound iron by Haemophilus species of human and porcine origins, FEMS Microbiol. Lett. 53 (1989) 123-127.

[22] Munch S., Grund S., Kruger M., Fimbriae and membranes on Haemophilus parasuis, Zentralbl. Veterinarmed. B 39 (1992) 59-64.

[23] Occhino D.A., Wyckoff E.E., Henderson D.P., Wrona T.J., Payne S.M., Vibrio cholerae iron transport: haem transport genes are linked to one of two sets of $\operatorname{ton} B, \operatorname{exb} B, \operatorname{exbD}$ genes, Mol. Microbiol. 29 (1998) 1493-1507.

[24] Rosner H., Kielstein P., Muller W., Rohrmann B., Relationship between serotype, virulence and SDS-PAGE protein patterns of Haemophilus parasuis, Dtsch. Tierarztl. Wochenschr. 98 (1991) 327-330.

[25] Ruiz A., Oliveira S., Torremorell M., Pijoan C. Outer membrane proteins and DNA profiles in strains of Haemophilus parasuis recovered from systemic and respiratory sites, J. Clin. Microbiol. 39 (2001) 1757-1762.

[26] Sambrook J., Fritsch E.F., Maniatis T., Molecular Cloning: A laboratory Manual, 2nd edition, Cold Spring Harbor Laboratory Press, New York, USA, 1989.

[27] Schaffer S., Hantke K., Braun V., Nucleotide sequence of the iron regulatory gene fur, Mol. Gen. Genet. 200 (1985) 110-113.

[28] Smart N.L., Miniats O.P., Preliminary assessment of a Haemophilus parasuis bacterin for use in specific pathogen free swine, Can. J. Vet. Res. 53 (1989) 390-393.

[29] Tonpitak W., Thiede S., Oswald W., Baltes N., Gerlach G.F., Actinobacillus pleuropneumoniae iron transport: a set of exbBD genes is transcriptionally linked to the $t b p B$ gene and required for utilization of transferrin-bound iron, Infect. Immun. 68 (2000) 1164-1170.

To access this journal online: www.edpsciences.org 